\title{
Ultrasound-promoted synthesis of novel dihydropyrido[2,3-d:5,6- d']dipyrimidine derivatives
}

Manouchehr Mamaghani ${ }^{a}{ }^{a}{ }_{*}$, Parivash Jahanshahi ${ }^{a}$, Ainaz Karimi ${ }^{a}$, and Roghayeh Hossein Nia ${ }^{b}$ ${ }^{a}$ Department of Chemistry, Faculty of Sciences, Islamic Azad University, Rasht Branch, P.O. Box 41335-3516, Rasht, Iran.

${ }^{b}$ Department of Chemistry, Faculty of Sciences, University of Guilan, P.O. Box 41335-1914, Rasht, Iran. Fax: +981333333262.

\section{ABSTRACT}

*Corresponding author:: mchem41@gmail.com , m-chem41@guilan.ac.ir

An efficient synthesis of dihydropyrido[2,3-d:5,6- $d$ ]pyrimidine derivatives was developed via one-pot three-component reaction of 6-amino-2-(alkylthio)-pyrimidine-4(3H) one and aryl aldehydes in the mixed solvent of glacial acetic acid and DMF $(1: 1, v: v)$ under ultrasonic irradiation. In this protocol novel fused dihydropyrido[2,3-d]pyrimidines were synthesized in high yields (80-97\%) and lower reaction times (5-25 min).

\section{Keywords}

6-amino-2-(alkylthio)pyrimidin-4(3H)-one; pyrido[2,3- $d$ ]pyrimidine; ultrasonic irradiation; three-component reaction.

\section{Council for Innovative Research}

Peer Review Research Publishing System

\section{Journal: Journal of Advances in Chemistry}

Vol.11, No.5

\section{editorjaconline@gmail.com}




\section{INTRODUCTION}

Pyridopyrimidines, their oxo and thioxo derivatives have been recognized as important organic materials in drug design, and showing interesting biological activities. For example they are known to be inhibitors of tyrosine kinase of the epidermal growth factor receptor family [1], antiviral [2], antioxidant [3] and antitumor [4]. Among them, pyrimido[4,5d]pyrimidines and pyrimido[2,3-d]pyrimidines, have been known to display a wide range of pharmacological activities [5,6]. It is well known that many pyrido[2,3-d]pyrimidine derivatives possess interesting biological logical properties. It has been proven that they possess diuretic [7], antibacterial [8], analgesic, anti-inflammatory [9], antiviral [10], anticonvulsive [11,12], antipyretic [13], cardiotonic [14, 15], antitumoral [16], bactericidal [17], bronchiodilator [18], antihistaminic [19], and other activities [20]. As a result, compounds of this class present considerable interest for research. Considering the important biological properties of fused pyrimidines, a number of methods have been reported for the synthesis of these heterocycles [21-28]. Some of these methods, suffer from one or more disadvantages such as using costly reagents, toxic catalysts and solvents, low yielding of products, harsh reaction conditions, and require prolonged heating and tedious work. As a result, development of a simple and green protocol for the synthesis of pyrido[2,3-d]pyrimidines is highly required.

On the other hand green chemistry has become a major driving force for organic chemists to develop eco-friendly routes for the synthesis of organic compounds. For example, the possibility of performing reactions under ultrasound irradiations. ultrasonic reactions have been increasingly used as green, clean and environmentally benign routes for the preparation of organic compounds [29-34]. A large number of organic reactions can be carried out in higher yield, shorter reaction time and under milder conditions, by using ultrasonic irradiation [35-38].

These abservations led us to attempt the synthesis of some novel pyridodipyrimidine derivatives using 6-amino-2(alkylthio)pyrimidin-4(3H)-one under ultrasonic irradiations.

\section{RESULTS AND DISCUSSION}

In continuation of our ongoing program aiming to develop efficient and eco-friendly methods for the synthesis of biologically important heterocyclic compounds [39-42], we carried out a simple one-pot three-component reaction reaction for the synthesis of dihydropyridopyrimidines under ultrasonic irradiation. In this protocol the reaction of 6-amino-2-(alkylthio)pyrimidin-4(3H)-one and arylaldehydes was studied in the mixed solvent of glacial acetic acide and DMF $(1: 1, \mathrm{v}: \mathrm{v})$ under ultrasonic irradiation (Scheme 1). The reaction furnished desired dihydropyridopyrimines in excellent yields (80-97\%) and short reaction times (5-25 min) (Table 3).

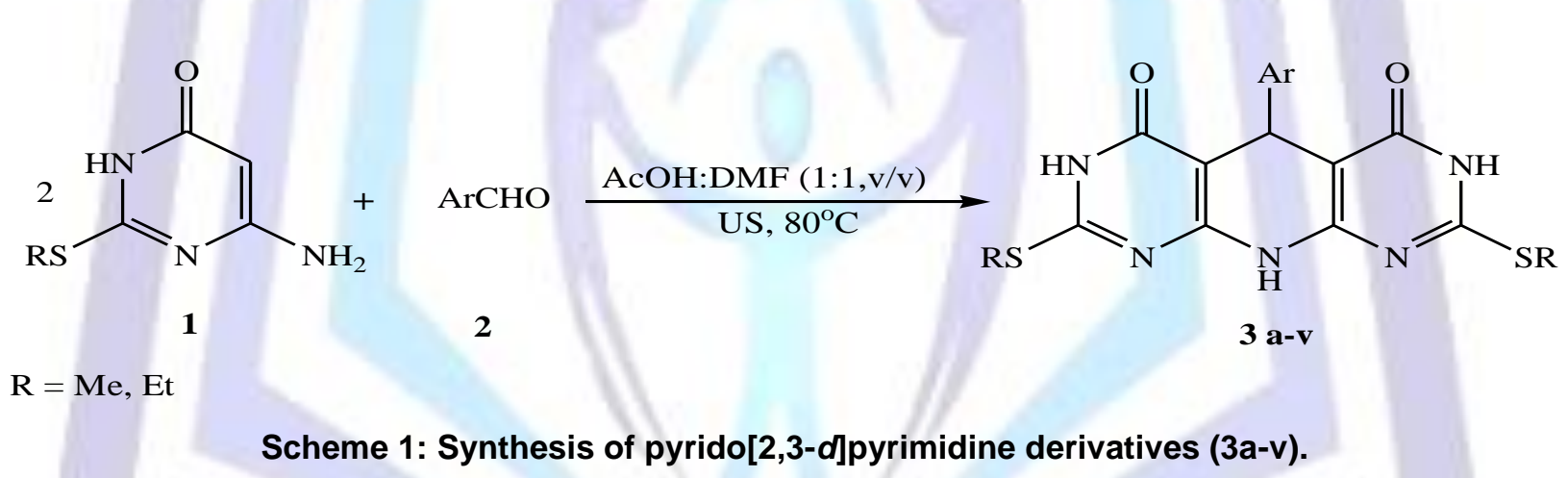

In the initial experiments, different solvents and catalysts screened for the synthesis of dihydropyridopyrimine derivatives (3a-v). To optimize the reaction conditions, preparation of 5-(4-chlorophenyl)-2,8-bis(ethylthio)-5,10-dihydropyrido[2,3d:5,6-d']dipyrimidin-4,6(3H,7H)-dione) (3a) by the reaction of 6-amino-2-(ethylthio)pyrimidin-4(3H)-one $(2 \mathrm{mmol})$ and 4 chlorobenzaldehyde $(1 \mathrm{mmol})$ was selected as a model reaction. The results are summarized in Table 1. It is evident from the results that $\mathrm{AcOH} / \mathrm{DMF}(1: 1, \mathrm{v}: \mathrm{v})$ is the most effective reaction medium among the solvents and catalysts selected, resulting in the highest yield $(97 \%)$ and the shortest reaction time $(10 \mathrm{~min})$ at $80^{\circ} \mathrm{C}$. For comparison the preparation of $3 a$ was carried out under conventional heating at reflux temperature which furnished the product at lower yield and much higher reaction time (Table 1, entry 1 ).

Table 1. Effect of various catalysts and solvents in the synthesis of $3 a$.

\begin{tabular}{|c|c|c|c|c|c|}
\hline \multirow{2}{*}{ Entry } & Catalyst/Solvent & \multicolumn{2}{|c|}{ Conventional Method $^{\text {a }}$} & \multicolumn{2}{|c|}{ Sonochemical method $^{\mathbf{b}}$} \\
\cline { 3 - 6 } & & Time (min) & Yield $^{\mathbf{c}}$ (\%) & Time (min) & Yield $^{\mathbf{c}}$ (\%) \\
\hline 1 & AcOH/DMF (1:1) & 120 & 76 & 10 & 97 \\
\hline 2 & AcOH/DMF (1:2) & 120 & 70 & 13 & 92 \\
\hline 3 & $P-T S A / E t O H$ & 180 & 72 & 15 & 85 \\
\hline 4 & AcOH & 210 & 75 & 15 & 87 \\
\hline 5 & AcOH/EtOH (1:1) & 220 & 72 & 20 & 80 \\
\hline 6 & Ethylene glycol & 210 & 60 & 25 & 70 \\
\hline
\end{tabular}




\begin{tabular}{|c|c|c|c|c|c|}
\hline 7 & $\mathrm{EtOH}$ & 250 & 60 & 25 & 72 \\
\hline
\end{tabular}

${ }^{\mathrm{a}}$ Reflux conditions.

${ }^{\mathrm{b}} \mathrm{At} 80^{\circ} \mathrm{C}$.

Isolated Yield.

To optimize the reaction condition under ultrasonic irradiations, we verified different temperature for the preparation of $3 a$ and the best result was obtained at $80^{\circ} \mathrm{C}$ (Table 2).

Table 2. Effect of various temperature in the synthesis of $3 a$.

\begin{tabular}{|c|c|c|c|}
\hline Entry & ${\text { Temperature }\left({ }^{\circ} \mathbf{C}\right)^{\text {a }}}^{\text {Time (min) }}$ & Yield (\%) \\
\hline 1 & 25 & 40 & 60 \\
\hline 2 & 60 & 15 & 83 \\
\hline 3 & 80 & 10 & 97 \\
\hline
\end{tabular}

The starting material 1 was synthesized by condensation of thiourea with ethylcyanoacetate in sodium ethoxide and followed by alkylation with alkyl iodide according to the known procedure [43]. By using the optimized reaction conditions several derivatives of dihydropyridopyrimines (3a-v) were prepared in high to excellent yields ( $80-97 \%)$ and lower reaction times (5-25 min) (Table 3). The structures of all the products were confirmed by spectroscopic (IR, ${ }^{1} \mathrm{H}$ NMR, ${ }^{13} \mathrm{C}$ NMR) and elemental analyses.

Table 3. Synthesis of 3a-v under optimized conditions.

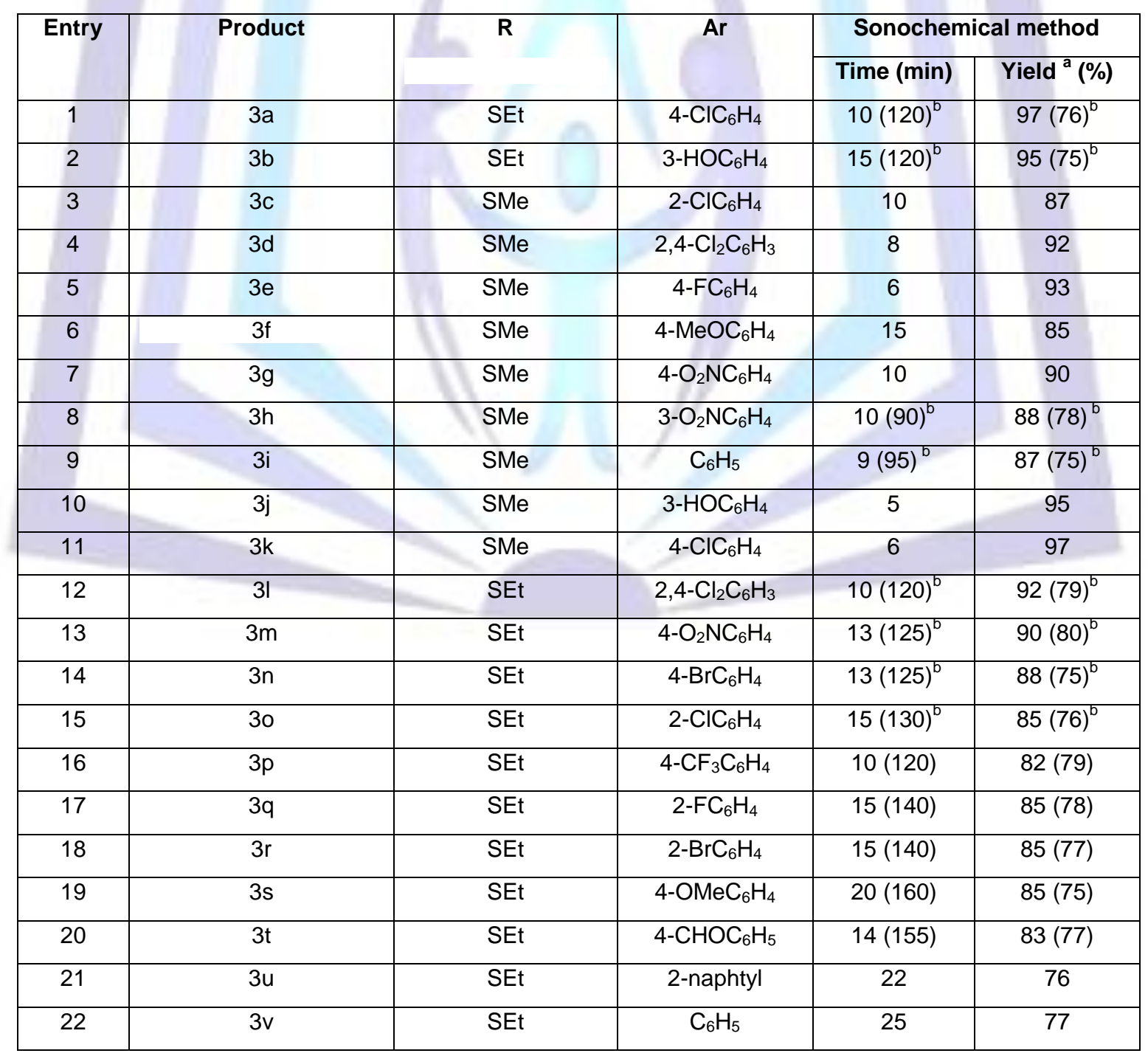


a Isolated Yield.

${ }^{\mathrm{b}}$ Conventional heating in refluxing $\mathrm{AcOH} / \mathrm{DMF}(1: 1)$.

The plausible mechanism of the formation of 3a-v is outlined in Scheme 2. Due to the protonation of aldehyde by $\mathrm{AcOH}$, aldehyde becomes a strong electrophile in reaction with 1 . The high reactivity of enamine 1 allows the addition of this molecule to the protonated aldehyde through a Knoevenagel condensation and producing the arylidene intermediate 5 by loss of $\mathrm{H}_{2} \mathrm{O}$. Finally Michael addition of second molecule of enamine 1 to the intermediate 5 and loss of $\mathrm{NH} 3$ furnishes the desired dihydropyridodipyrimidines 4 (Scheme 2).<smiles>[Y17]C=c1c(=[NH2+])nc([R5])c(=CC(=O)O)[nH]c1=O</smiles><smiles>[R7]c1nc2c(c(=O)[nH]1)C([Y19])c1c(nc([R8])[nH]c1=O)N2</smiles>

$3 \mathrm{a}-\mathrm{v}$

Scheme 2: Plausible mechanism for the synthesis of (3a-v).

\section{EXPERIMENTAL}

Melting points were recorded on a Büchi B-545 apparatus in open capillary tubes. IR spectra were determined on a Shimadzo IR-470 spectrometer. ${ }^{1} \mathrm{H}$ NMR and ${ }^{13} \mathrm{C}$ NMR spectra were recorded on a $400 \mathrm{MHz}$ Bruker DRX-400 and DMSO- $\mathrm{d}_{6}$ as solvent and TMS as an internal standard. Elemental analyses were done on a Carlo-Erba EA1110CNNO-S analyzer and agreed with the calculated values. Chemicals were purchased from Merck and Fluka. All solvents used were dried and distilled according to standard procedures.

\section{General procedure for preparation of (3a-v)}

A mixture of 6 -amino-2-(alkylthio)pyrimidin-4(3H)-one $(2 \mathrm{mmol})$ and aldehyde $(1 \mathrm{mmol})$ in AcOH/DMF(1:1, v:v) $(6 \mathrm{~mL})$ was heated under reflux or by ultrasonic irradiations using Elmasonic $\mathrm{S}-40 \mathrm{H}$ ultrasonic cleaning unit at $80{ }^{\circ} \mathrm{C}$ (under silent condition). The progress of the reaction was monitored by TLC (EtOAc/hexane/methanol 8:3:1). After completion of the reaction, the mixture was poured into ice-water, the solid obtained was filtered off, washed by THF and recrystalyzed from $\mathrm{DMF} / \mathrm{H}_{2} \mathrm{O}$ to furnish the desired pure products.

5-(4-Chlorophenyl)-2,8-bis(ethylthio)-5,10-dihydropyrido[2,3-d:5,6-d ]dipyrimidin-4,6(3H,7H)-dione (3a). White powder, m.p. > $300^{\circ} \mathrm{C}$. IR, v/cm ${ }^{-1}$ : 3354; 3180; 3033; 2967; 2933; 2866; 1653; 1610; 1558; 1446; 1446; 1335; 1236; 1094; 831. ${ }^{1} \mathrm{H}$ NMR (DMSO-d $)$ ), $\delta: 1.31\left(\mathrm{t}, \mathrm{J}=6.9 \mathrm{~Hz}, 6 \mathrm{H}, \mathrm{CH}_{3}\right) ; 3.11\left(\mathrm{q}, 4 \mathrm{H}, \mathrm{CH}_{2}, J=6.9 \mathrm{~Hz}\right) ; 5.45(\mathrm{~s}, 1 \mathrm{H}, \mathrm{CH}) ; 6.82(\mathrm{br} . \mathrm{s}, 1 \mathrm{H}$, $\mathrm{NH}) ; 7.04(\mathrm{~d}, 2 \mathrm{H}, \mathrm{Ar}-\mathrm{H}, J=8.0 \mathrm{~Hz}) ; 7.26(\mathrm{~d}, 2 \mathrm{H}, \mathrm{Ar}-\mathrm{H}, J=8.0 \mathrm{~Hz}) ; 11.98(\mathrm{br} . \mathrm{s}, 2 \mathrm{H}, \mathrm{NH}-\mathrm{C}=\mathrm{O}) .{ }^{13} \mathrm{C}$ NMR $\left(\mathrm{DMSO}-\mathrm{d}_{6}\right), \delta_{\mathrm{C}}$ : 
15.2; 24.3; 33.5; 92.7; 128.0; 129.0; 129.8; 138.8; 158.3; 161.9; 163.8. Anal. Cald. for $\mathrm{C}_{19} \mathrm{H}_{18} \mathrm{CIN}_{5} \mathrm{O}_{2} \mathrm{~S}_{2}(447.96)$. Calculated (\%): C, 50.94; H, 4.05; N, 15.63; Found (\%): C, 50.86; H, 4.16; N, 15.79.

5-(3-Hydroxyphenyl)-2,8-bis(ethylthio)-5,10-dihydropyrido[2,3-d:5,6- $d^{\prime}$ ]dipyrimidin-4,6(3H,7H)-dion (3b). White powder, m.p. > $300^{\circ} \mathrm{C}$. IR, v/cm ${ }^{-1}: 3441 ; 3340 ; 3155 ; 2968 ; 2926 ; 2862 ; 1647 ; 1616 ; 1558 ; 1452 ; 1452 ; 1367 ; 1230 ; 878$; 812; 698. ${ }^{1} \mathrm{H}$ NMR (DMSO-d $\left.)_{6}\right), \delta: 1.31\left(\mathrm{t}, 6 \mathrm{H}, \mathrm{CH}_{3}, J=7.0 \mathrm{~Hz}\right) ; 3.11\left(\mathrm{q}, 4 \mathrm{H}, \mathrm{CH}_{2}, J=7.0 \mathrm{~Hz}\right) ; 5.41(\mathrm{~s}, 1 \mathrm{H}, \mathrm{CH}) ; 6.50(\mathrm{~m}$, $3 \mathrm{H}, \mathrm{Ar}-\mathrm{H}$ ); 6.78 (br. s, $1 \mathrm{H}, \mathrm{NH}) ; 6.98(\mathrm{t}, 1 \mathrm{H}, \mathrm{Ar}-\mathrm{H}, J=7.6 \mathrm{~Hz}) ; 9.0$ (s, $1 \mathrm{H}, \mathrm{OH}) ; 11.93$ (br. s, $2 \mathrm{H}, \mathrm{NH}-\mathrm{C}=\mathrm{O}) .{ }^{13} \mathrm{C} \mathrm{NMR}$ $\left(\right.$ DMSO-d ${ }_{6}$ ), $\delta_{\mathrm{C}}: 15.2 ; 24.3 ; 33.8 ; 112.2 ; 112.3 ; 113.9 ; 114.0 ; 117.9 ; 128.9 ; 141.4 ; 157.3 ; 157.4 ; 157.8$. Anal. Cald. for $\mathrm{C}_{19} \mathrm{H}_{19} \mathrm{~N}_{5} \mathrm{O}_{3} \mathrm{~S}_{2}$ (429.52). Calculated (\%): C, 53.13; H, 4.46; N, 16.31; Found (\%): C, 53.00; H, 4.59; N, 16.18.

5-(2-Chlorophenyl)-2,8-bis(methylthio)-5,10-dihydropyrido[2,3-d:5,6- $d$ ] dipyrimidin-4,6(3H,7H)-dione (3c). White powder, m.p. $>300{ }^{\circ} \mathrm{C} . \mathrm{IR}, \mathrm{v} / \mathrm{cm}^{-1}: 3427 ; 3209 ; 3112 ; 2923 ; 1649 ; 1608 ; 1537 ; 1431 ; 1344 ; 1238 ; 1033 ; 771 ; 739 .{ }^{1} \mathrm{H}$ NMR (DMSO-d $\mathrm{d}_{6}$ ), $\delta: 2.47$ (s, 6H, CH 3 ); 5.42 (s, 1H, CH); 6.88 (br.s, 1H, NH); 7.27-7.13 (m, 4H, Ar-H); 11.94 (br. s, $2 \mathrm{H}$, $\mathrm{NH}-\mathrm{C}=\mathrm{O}$ ). ${ }^{13} \mathrm{C}$ NMR (DMSO-d6), $\delta_{\mathrm{c}}: 12.9 ; 33.3 ; 110.8 ; 126.8 ; 127.4 ; 127.5 ; 129.3 ; 129.6 ; 133.1 ; 138.7 ; 158.6 ; 161.5$. Anal. Cald. for $\mathrm{C}_{17} \mathrm{H}_{14} \mathrm{CIN}_{5} \mathrm{O}_{2} \mathrm{~S}_{2}$ (419.91). Calculated (\%): C, 48.63; H, 3.36; N, 16.68; Found, \%: C, 48.50; H, 3.16; N, 16.49 .

5-(2,4-Dichlorophenyl)-2,8-bis(methylthio)-5,10-dihydropyrido[2,3-d:5,6-d ]dipyrimidin-4,6(3H,7H)-dione (3d). White powder, m.p. $>300{ }^{\circ} \mathrm{C}$. IR spectrum, v/cm ${ }^{-1}: 3360 ; 3200 ; 3009 ; 2926 ; 2813 ; 1658 ; 1607 ; 1577 ; 1539 ; 1437 ; 1437 ; 1340 ;$ 1236; 1099; 1043; 890; 808; 758. ${ }^{1} \mathrm{H}$ NMR (DMSO-d $)$, ס: 2.47 (s, 6H, CH $)$; $5.38(\mathrm{~s}, 1 \mathrm{H}, \mathrm{CH}) ; 6.94$ (br. s, $\left.1 \mathrm{H}, \mathrm{NH}\right) ; 7.21$ (d, $1 \mathrm{H}, \mathrm{Ar}-\mathrm{H}, J=8.8 \mathrm{~Hz}) ; 7.32(\mathrm{dd}, 2.1 \mathrm{~Hz}, 1 \mathrm{H}, \mathrm{Ar}-\mathrm{H}, J=8.6 \mathrm{~Hz}) ; 7.41$ (d, $1 \mathrm{H}, \mathrm{Ar}-\mathrm{H}, J=2.0 \mathrm{~Hz}) ; 12.00$ (br. s, 2H, NH-C=O) ${ }^{13} \mathrm{C}$ NMR (DMSO-d ${ }_{6}$ ), $\delta_{C}: 12.9 ; 33.0 ; 92.3 ; 127.0 ; 129.0 ; 130.6 ; 131.0 ; 133.9 ; 138.0 ; 158.9 ; 161.4,161.5 ; 161.6 ; 163.2$. Anal. Cald. for $\mathrm{C}_{17} \mathrm{H}_{13} \mathrm{Cl}_{2} \mathrm{~N}_{5} \mathrm{O}_{2} \mathrm{~S}_{2}$ (454.36). Calculated (\%): C, 44.94; H, 2.88; N, 15.41; Found (\%): C, 44.82; H, 2.97; N, 15.25 .

5-(4-Fluorophenyl)-2,8-bis(methylthio)-5,10-dihydropyrido[2,3-d:5,6- $d$ ' ]dipyrimidin-4,6(3H,7H)-dione (3e). White powder, m.p. $>300{ }^{\circ} \mathrm{C}$. IR, v/cm ${ }^{-1}$ : 3456; 3292; 3163; 3090; 2999; 2922; 1645; 1614; 1577; 1450; 1450; 1335; 1234; 1155; 846. ${ }^{1} \mathrm{H}$ NMR (DMSO-d $\mathrm{d}_{6}$ ), $\delta: 2.48\left(\mathrm{~s}, 6 \mathrm{H}, \mathrm{CH}_{3}\right) ; 5.44$ (s, $\left.1 \mathrm{H}, \mathrm{CH}\right) ; 6.81$ (br.s, $\left.1 \mathrm{H}, \mathrm{NH}\right) ; 7.05-6.98$ (m, 4H, Ar-H); 12.01 (br. s, $2 \mathrm{H}, \mathrm{NH}-\mathrm{C}=\mathrm{O}) .{ }^{13} \mathrm{C}$ NMR (DMSO-d $), \delta_{\mathrm{C}}: 12.9 ; 33.3 ; 92.9 ; 114.6\left(\mathrm{~d},{ }^{2} \mathrm{~J}_{\mathrm{C}-\mathrm{F}}=21.0 \mathrm{~Hz}\right) ; 128.7\left(\mathrm{~d},{ }^{3} \mathrm{~J}_{\mathrm{C}-\mathrm{F}}=8.0 \mathrm{~Hz}\right) ; 135.5\left(\mathrm{~d},{ }^{4} \mathrm{~J}_{\mathrm{C}}\right.$ $\mathrm{F}=2.0 \mathrm{~Hz}) ; 158.8 ; 160.6\left(\mathrm{~d},{ }^{1} \mathrm{~J}_{\mathrm{C}-\mathrm{F}}=239.0 \mathrm{~Hz}\right) ; 163.7$. Anal. Cald. for $\mathrm{C}_{17} \mathrm{H}_{14} \mathrm{FN}_{5} \mathrm{O}_{2} \mathrm{~S}_{2}(403.45)$. Calculated $(\%): \mathrm{C}, 50.61 ; \mathrm{H}$, $3.50 ; \mathrm{N}, 17.36$; Found (\%): C, 50.48; H, 3.55; N, 17.47 .

5-(4-Methoxyphenyl)-2,8-bis(methylthio)-5,10-dihydropyrido[2,3-d:5,6- $d$ ] dipyrimidin-4,6(3H,7H)-dion (3f). White powder, m.p. > $300^{\circ} \mathrm{C}$. IR, v/cm ${ }^{-1}: 3400 ; 3312 ; 3180 ; 3080 ; 2995 ; 2926 ; 1633 ; 1610 ; 1577 ; 1541 ; 1512 ; 1442 ; 1442 ; 1338 ;$ 1240; 1034; 831. ${ }^{1} \mathrm{H}$ NMR (DMSO-d $)$, $\delta: 2.48\left(\mathrm{~s}, 6 \mathrm{H}, \mathrm{CH}_{3}\right) ; 3.71\left(\mathrm{~s}, 3 \mathrm{H}, \mathrm{OCH}_{3}\right) ; 5.42(\mathrm{~s}, 1 \mathrm{H}, \mathrm{CH}) ; 6.77(\mathrm{~d}, 2 \mathrm{H}, \mathrm{Ar}-\mathrm{H}, \mathrm{J}=$ $8.4 \mathrm{~Hz}$ ); 6.92 (d, $2 \mathrm{H}, \mathrm{Ar}-\mathrm{H}, \mathrm{J}=8.4 \mathrm{~Hz}$ ); 7.26 (br. s, $1 \mathrm{H}, \mathrm{NH}) ; 11.96$ (br. s, $2 \mathrm{H}, \mathrm{NH}-\mathrm{C}=\mathrm{O}) .{ }^{13} \mathrm{C}$ NMR (DMSO-d 6 ), $\delta_{\mathrm{c}}: 12.9$; 33.2; 55.3; 93.3; 113.5;128.0; 131.3; 157.3; 158.6; 161.9; 163.7. Anal. Cald. for Calculated (\%): C, 52.03; H, 4.12; N, 16.86; Found (\%): C, 52.20; $\mathrm{H}, 4.02 ; \mathrm{N}, 16.98 . \mathrm{C}_{18} \mathrm{H}_{17} \mathrm{~N}_{5} \mathrm{O}_{3} \mathrm{~S}_{2}(415.49)$.

5-(4-Nitrophenyl)-2,8-bis(methylthio)-5,10-dihydropyrido[2,3-d:5,6-d ]dipyrimidin-4,6(3H,7H)-dione (3g). White powder, m.p. > $300{ }^{\circ} \mathrm{C}$. IR, v/cm ${ }^{-1}: 3447 ; 3300 ; 3188 ; 2928 ; 2851 ; 1642 ; 1610 ; 1533 ; 1515 ; 1438 ; 1515 ; 1342 ; 1438 ; 1342 ;$

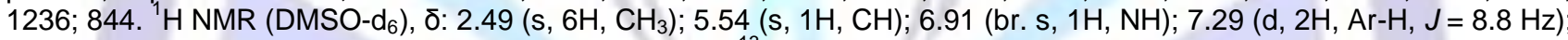
$8.10(\mathrm{~d}, 2 \mathrm{H}, \mathrm{Ar}-\mathrm{H}, J=8.8 \mathrm{~Hz}) ; 12.08(\mathrm{~s}, 2 \mathrm{H}, \mathrm{NH}-\mathrm{C}=\mathrm{O}) .{ }^{13} \mathrm{C}$ NMR (DMSO-d $\left.\mathrm{d}_{6}\right), \delta_{\mathrm{c}}: 12.9 ; 34.4 ; 92.4 ; 123.4 ; 128.4 ; 145.7 ;$ 149.0; 159.2; 163.0. Anal. Cald. for $\mathrm{C}_{17} \mathrm{H}_{14} \mathrm{~N}_{6} \mathrm{O}_{4} \mathrm{~S}_{2}$ (430.46). Calculated (\%): C, 47.43; H, 3.28; N, 19.52; Found (\%): C 47.58; H, 3.12; N, 19.41.

5-(3-Nitrophenyl)-2,8-bis(methylthio)-5,10-dihydropyrido[2,3-d:5,6- $d^{d}$ ]dipyrimidin-4,6(3H,7H)-dione (3h). White powder, m.p. $>300^{\circ} \mathrm{C}$. IR, v/cm ${ }^{-1}: 3355 ; 3198 ; 2923 ; 2850 ; 1658 ; 1606 ; 1528 ; 1434 ; 1523 ; 1342 ; 1342 ; 1238 ; 831 .{ }^{1} \mathrm{H}$ NMR (DMSO-d $\left.\mathrm{d}_{6}\right), \delta: 2.50\left(\mathrm{~s}, 6 \mathrm{H}, \mathrm{CH}_{3}\right) ; 5.56(\mathrm{~s}, 1 \mathrm{H}, \mathrm{CH}) ; 6.94$ (br. s, $\left.1 \mathrm{H}, \mathrm{NH}\right) ; 7.55-7.47(\mathrm{~m}, 2 \mathrm{H}, \mathrm{Ar}-\mathrm{H}) ; 7.80(\mathrm{dd}, 1 \mathrm{H}, \mathrm{Ar}-\mathrm{H}$, $J_{1}=3.2 \mathrm{~Hz}, J_{2}=2.0 \mathrm{~Hz}$ ); 8.03-8.00 (m, $\left.1 \mathrm{H}, \mathrm{Ar}-\mathrm{H}\right) ; 12.11$ (br. s, $\left.2 \mathrm{H}, \mathrm{NH}-\mathrm{C}=\mathrm{O}\right) .{ }^{13} \mathrm{C}$ NMR (DMSO-d $)$ ), $\delta_{\mathrm{c}}: 12.9 ; 33.9 ; 92.4 ;$ 120.8; 121.7; 129.6; 134.3; 142.5; 148.2; 159.2; 163.0. $\mathrm{C}_{17} \mathrm{H}_{14} \mathrm{~N}_{6} \mathrm{O}_{4} \mathrm{~S}_{2}$ (430.46). Anal. Cald. for Calculated (\%): C, 47.43; H, 3.28; N, 19.52; Found (\%): C, 47.31; H, 3.39; N, 19.32.

5-(Phenyl)-2,8-bis(methylthio)-5,10-dihydropyrido[2,3-d:5,6- $d$ ] dipyrimidin-4,6(3H,7H)-dione (3i). White powder, m.p. $>300{ }^{\circ} \mathrm{C}$. IR, v/cm ${ }^{-1}$ : 3448; 3290; 3159; 3084; 2997; 2916; 2878; 1641; 1610; 1578; 1531; 1450; 1450; 1326; 1236; 771; 692. ${ }^{1} \mathrm{H}$ NMR (DMSO-d $\left.\mathrm{d}_{6}\right), \delta: 2.48\left(\mathrm{~s}, 6 \mathrm{H}, \mathrm{CH}_{3}\right) ; 5.47(\mathrm{~s}, 1 \mathrm{H}, \mathrm{CH}) ; 6.08$ (br. s, $\left.1 \mathrm{H}, \mathrm{NH}\right) ; 7.02(\mathrm{~d}, 2 \mathrm{H}, \mathrm{Ar}-\mathrm{H}, \mathrm{J}=8.4 \mathrm{~Hz}) ; 7.1$ (dt, $1 \mathrm{H}, \mathrm{Ar}-\mathrm{H}, J_{1}=7.3, J_{2}=1.0 \mathrm{~Hz}$ ); 7.20 (t, $\left.2 \mathrm{H}, \mathrm{Ar}-\mathrm{H}, J=7.4 \mathrm{~Hz}\right) ; 11.98$ (br. s, $\left.2 \mathrm{H}, \mathrm{NH}-\mathrm{C}=\mathrm{O}\right) .{ }^{13} \mathrm{C}$ NMR (DMSO-d $)$ ), $\delta_{\mathrm{C}}$ : 12.9; 33.9; 93.0; 125.3; 127.0; 128.1; 139.7; 158.1; 161.2; 163.0. Anal. Cald. for $\mathrm{C}_{17} \mathrm{H}_{15} \mathrm{~N}_{5} \mathrm{O}_{2} \mathrm{~S}_{2}$ (385.46). Calculated (\%): C, 52.97; H, 3.92; N, 18.17; Found (\%): C, 52.81; H, 3.98; N, 18.05.

5-(3-Hydroxyphenyl)-2,8-bis(methylthio)-5,10-dihydropyrido[2,3-d:5,6- $d$ ' ]dipyrimidin-4,6(3H,7H)-dione (3j). White powder, m.p. > $300^{\circ} \mathrm{C}$. IR, v/cm ${ }^{-1}$ : 3446; 3294; 3178; 3088; 2995; 2920; 2891; 1641; 1606; 1577; 1448; 1448; 1336; 1234; 820; 783; 690. ${ }^{1} \mathrm{H}$ NMR (DMSO-d $)$ ), $\delta: 2.43\left(\mathrm{~s}, 6 \mathrm{H}, \mathrm{CH}_{3}\right) ; 5.37(\mathrm{~s}, 1 \mathrm{H}, \mathrm{CH}) ; 6.43(\mathrm{~m}, 3 \mathrm{H}, \mathrm{Ar}-\mathrm{H}) ; 6.73$ (br. s, $\left.1 \mathrm{H}, \mathrm{NH}\right) ; 6.93(\mathrm{t}$, $1 \mathrm{H}, \mathrm{Ar}-\mathrm{H}, J=7.58 \mathrm{~Hz}$ ); 8.92 (br. s, $1 \mathrm{H}, \mathrm{OH}$ ); 11.90 (br. s, NH-C=O). ${ }^{13} \mathrm{C} N M R$ (DMSO-d $)$ ), $\delta_{c}: 12.9 ; 33.8 ; 112.2 ; 113.8 ;$ $113.9 ; 117.9 ; 128.9 ; 141.4 ; 157.3 ; 157.4 ; 158.6 ; 162.8$. Anal. Cald. for $\mathrm{C}_{17} \mathrm{H}_{15} \mathrm{~N}_{5} \mathrm{O}_{3} \mathrm{~S}_{2}$ (401.46). Calculated (\%): C, 50.86; H, 3.77; N, 17.44; Found (\%): C, 50.60; H, 3.64; N, 17.35 . 
5-(4-Chlorophenyl)-2,8-bis(methylthio)-5,10-dihydropyrido[2,3-d:5,6- $d$ ] dipyrimidin-4,6(3H,7H)-dione (3k). White powder, m.p. > $300{ }^{\circ} \mathrm{C} . \mathrm{IR}, \mathrm{v} / \mathrm{cm}^{-1}: 3445 ; 3391 ; 3306 ; 3173 ; 3091 ; 2923 ; 1635 ; 1608 ; 1577 ; 1539 ; 1442 ; 1442 ; 1336 ;$ 1240; 1091; 829. ${ }^{1} \mathrm{H}$ NMR (DMSO-d $), \delta: 2.43$ (s, 6H, $\left.\mathrm{CH}_{3}\right) ; 5.40$ (s, 1H, CH); 6.76 (br. s, 1H, NH); $6.99(\mathrm{~d}, 2 \mathrm{H}, \mathrm{Ar}-\mathrm{H}, \mathrm{J}=$ $8.32 \mathrm{~Hz}$ ); 7.22 (d, $2 \mathrm{H}, \mathrm{Ar}-\mathrm{H}, J=8.32 \mathrm{~Hz}$ ); 11.96 (br. s, $2 \mathrm{H}, \mathrm{NH}-\mathrm{C}=\mathrm{O}$ ). ${ }^{13} \mathrm{C}$ NMR (DMSO-d $)_{6}$ ), $\delta_{\mathrm{c}}: 12.9 ; 33.6 ; 92.7 ; 128.0 ;$ $129.0 ; 129.8 ; 138.9 ; 159.0 ; 162.3 ; 163.8$. Anal. Cald. for $\mathrm{C}_{17} \mathrm{H}_{14} \mathrm{CIN}_{5} \mathrm{O}_{2} \mathrm{~S}_{2}$ (419.91). Calculated (\%): $\mathrm{C}, 48.63 ; \mathrm{H}, 3.36 ; \mathrm{N}$, 16.68; Found (\%): C, 48.68; H, 3.25; N, 16.52 .

5-(2,4-Dichlorophenyl)-2,8-bis(ethylthio)-5,10-dihydropyrido[2,3-d:5,6- $d$ ' ]dipyrimidin-4,6(3H,7H)-dione (3I). White powder, m.p. > $300^{\circ} \mathrm{C}$. IR, v/cm ${ }^{-1}$ : 3360; 3205; 2963; 2924; 2880; 1655; 1605; 1558; 1441; 1441; 1370; 1240; 1133; 1043; 874; 690. ${ }^{1} \mathrm{H}$ NMR (DMSO-d $)_{6}$ ), $\delta: 1.30$ (t, $6 \mathrm{H}, \mathrm{CH}_{3}, J=7.4 \mathrm{~Hz}$ ); 3.10 (q, $4 \mathrm{H}, \mathrm{CH}_{2}, J=7.4 \mathrm{~Hz}$ ); 5.39 (s, $\left.1 \mathrm{H}, \mathrm{CH}\right) ; 6.89$ (br. s, $1 \mathrm{H}, \mathrm{NH}) ; 7.22(\mathrm{~d}, 1 \mathrm{H}, \mathrm{Ar}-\mathrm{H}, J=8.4 \mathrm{~Hz}) ; 7.35\left(\mathrm{dd}, 1 \mathrm{H}, \mathrm{Ar}-\mathrm{H}, J_{1}=8.4 \mathrm{~Hz}, J_{2}=2.4 \mathrm{~Hz}\right) ; 7.42(\mathrm{~d}, 1 \mathrm{H}, \mathrm{Ar}-\mathrm{H}, J=2.4 \mathrm{~Hz}) ; 11.88$ (br. s, $1 \mathrm{H}, \mathrm{NH}-\mathrm{C}=\mathrm{O}$ ); 12.07 (br. s, $1 \mathrm{H}, \mathrm{NH}-\mathrm{C}=\mathrm{O}$ ). ${ }^{13} \mathrm{C}$ NMR (DMSO-d ${ }_{6}$ ), $\delta_{\mathrm{C}}: 15.1 ; 24.3 ; 33.0 ; 127.0 ; 129.0 ; 130.6 ; 131.0 ;$ 133.9; 138.0; 158.2; 161.5. Anal. Cald. for $\mathrm{C}_{19} \mathrm{H}_{17} \mathrm{Cl}_{2} \mathrm{~N}_{5} \mathrm{O}_{2} \mathrm{~S}_{2}$ (482.41). Calculated (\%): C, 47.31; H, 3.55; N, 14.52; Found (\%): C, 47.20; H, 3.64; N, 14.60.

5-(4-Nitrophenyl)-2,8-bis(ethylthio)-5,10-dihydropyrido[2,3-d:5,6-d ]dipyrimidin-4,6(3H,7H)-dione (3m). Cream powder, m.p. > $300{ }^{\circ} \mathrm{C} . \mathrm{IR}, \mathrm{v} / \mathrm{cm}^{-1}: 3352 ; 3182 ; 2962 ; 2926 ; 2870 ; 1653 ; 1606 ; 1518 ; 1445 ; 1518 ; 1342 ; 1342 ; 1236 ; 845$. ${ }^{1} \mathrm{H}$ NMR (DMSO-d $)$, $\delta: 1.31\left(\mathrm{t}, 6 \mathrm{H}, \mathrm{CH}_{3}, J=7.4 \mathrm{~Hz}\right) ; 3.12\left(\mathrm{~m}, 4 \mathrm{H}, \mathrm{CH}_{2}\right) ; 5.55(\mathrm{~s}, 1 \mathrm{H}, \mathrm{CH}) ; 6.92(\mathrm{br} . \mathrm{s}, 1 \mathrm{H}, \mathrm{NH}) ; 7.30(\mathrm{~d}$, $2 \mathrm{H}, \mathrm{Ar}-\mathrm{H}, J=8.6 \mathrm{~Hz}$ ); $8.11(\mathrm{~d}, 2 \mathrm{H}, \mathrm{Ar}-\mathrm{H}, J=8.6 \mathrm{~Hz}) ; 12.07(\mathrm{~s}, 2 \mathrm{H}, \mathrm{NH}-\mathrm{C}=\mathrm{O}) .{ }^{13} \mathrm{C} N M R$ (DMSO-d 6 ), $\delta_{\mathrm{c}}: 15.2 ; 24.3 ; 34.5 ;$ 92.4; 123.4; 128.4; 145.7; 149.0; 158.6; 162.0; 163.9. Anal. Cald. for $\mathrm{C}_{19} \mathrm{H}_{18} \mathrm{~N}_{6} \mathrm{O}_{4} \mathrm{~S}_{2}(458.51)$. Calculated (\%): $\mathrm{C}, 49.77 ; \mathrm{H}$, 3.96; N, 18.33; Found (\%): C, 49.65; H, 3.84; N, 18.48

5-(4-Bromophenyl)-2,8-bis(ethylthio)-5,10-dihydropyrido[2,3-d:5,6-d ]dipyrimidin-4,6(3H,7H)-dion (3n). White powder, m.p. > $300^{\circ} \mathrm{C}$. IR, v/cm ${ }^{-1}: 3348 ; 3182 ; 2966 ; 2926 ; 2862 ; 1657 ; 1607 ; 1564 ; 1539 ; 1443 ; 1443 ; 1335 ; 1236 ; 1066$; 827. ${ }^{13} \mathrm{C}$ NMR (DMSO-d $)$, $\delta: 15.2 ; 24.3 ; 33.6 ; 92.6 ; 118.3 ; 129.5 ; 130.9 ; 139.4 ; 158.3 ; 162.1 ; 163.8 . \mathrm{C}_{19} \mathrm{H}_{18} \mathrm{BrN}_{5} \mathrm{O}_{2} \mathrm{~S}_{2}$ (492.41). Anal. Cald. for Calculated (\%): C, 46.34; H, 3.68; N, 14.22; Found (\%): C, 46.39; H, 3.52; N, 14.10.

5-(2-Chlorophenyl)-2,8-bis(ethylthio)-5,10-dihydropyrido[2,3-d:5,6- $d$ ] dipyrimidin-4,6(3H,7H)-dione (30). White powder, m.p. $>300^{\circ} \mathrm{C}$. IR, v/cm ${ }^{-1}: 3350 ; 3196 ; 2965 ; 2933 ; 1653 ; 1605 ; 1558 ; 1439 ; 1439 ; 1358 ; 1234 ; 1126 ; 1038,779$. ${ }^{1} \mathrm{H}$ NMR (DMSO-d $)$ ), $\delta: 1.30$ (t, $\left.6 \mathrm{H}, \mathrm{CH}_{3}, \mathrm{~J}=7.2 \mathrm{~Hz}\right) ; 3.11\left(\mathrm{q}, 4 \mathrm{H}, \mathrm{CH}_{2}, \mathrm{~J}=7.2 \mathrm{~Hz}\right) ; 5.43(\mathrm{~s}, 1 \mathrm{H}, \mathrm{CH}) ; 7.24-7.17(\mathrm{~m}, 3 \mathrm{H}, \mathrm{Ar}-$ $\mathrm{H}) ; 7.29$ (d, $1 \mathrm{H}, \mathrm{Ar}-\mathrm{H}, \mathrm{J}=7.6 \mathrm{~Hz}) ; 11.96$ (br. s, $2 \mathrm{H}, \mathrm{NH}-\mathrm{C}=\mathrm{O}) .{ }^{13} \mathrm{C}$ NMR (DMSO-d 6 ), $\mathrm{\delta}_{\mathrm{c}}: 15.1 ; 24.3 ; 33.3 ; 92.6 ; 126.9 ;$ $127.5 ; 129.3 ; 129.6 ; 133.1 ; 138.7 ; 158.0 ; 161.6 ; 161.7$. Anal. Cald. for $\mathrm{C}_{19} \mathrm{H}_{18} \mathrm{ClN}_{5} \mathrm{O}_{2} \mathrm{~S}_{2}(447.96)$. Calculated (\%): C, $50.94 ; \mathrm{H}, 4.05 ; \mathrm{N}, 15.63$; Found (\%): C, 50.76; H, 4.16; N, 15.44

5-(4-(Trifluoromethyl)phenyl)-2,8-bis(ethylthio)-5,10-dihydropyrido[2,3-d:5,6- $d$ ] dipyrimidin-4,6(3H,7H)-dion (3p). White powder, m.p. > $300{ }^{\circ} \mathrm{C}$. IR, v/cm ${ }^{-1}: 3348 ; 3184 ; 2960 ; 2940 ; 2867 ; 1653 ; 1610 ; 1541 ; 1448 ; 1448 ; 1327 ; 1163 ;$ 1119; 1238; 841. 'H NMR (DMSO-d $)$ ), $\delta: 1.31\left(\mathrm{t}, 6 \mathrm{H}, \mathrm{CH}_{3}, \mathrm{~J}=7.4 \mathrm{~Hz}\right) ; 3.12\left(\mathrm{~m}, 4 \mathrm{H}, \mathrm{CH}_{2}\right) ; 5.53$ (s, $\left.1 \mathrm{H}, \mathrm{CH}\right) ; 6.86$ (br. s, , $1 \mathrm{H}, \mathrm{NH}) ; 7.24(\mathrm{~d}, 2 \mathrm{H}, \mathrm{Ar}-\mathrm{H}, J=8.2 \mathrm{~Hz}) ; 7.57$ (d, $2 \mathrm{H}, \mathrm{Ar}-\mathrm{H}, \mathrm{J}=8.2 \mathrm{~Hz}) ; 12.03$ (br. s, $2 \mathrm{H}, \mathrm{NH}-\mathrm{C}=\mathrm{O}) .{ }^{13} \mathrm{C} N M R\left(D M S O-\mathrm{d}_{6}\right)$, $\delta_{\mathrm{C}}: 15.2 ; 24.3 ; 34.1 ; 92.6 ; 124.1\left(\mathrm{~d},{ }^{1} \mathrm{~J}_{\mathrm{C}-\mathrm{F}}=270.0 \mathrm{~Hz}, \mathrm{CF}_{3}\right) ; 125.0\left(\mathrm{q},{ }^{3} \mathrm{~J}_{\mathrm{C}-\mathrm{F}}=3.0 \mathrm{~Hz}\right.$ ); $126.1\left(\mathrm{q},{ }^{2} \mathrm{~J}_{\mathrm{C}-\mathrm{F}}=31.0 \mathrm{~Hz}, \mathrm{C}-\mathrm{CF}_{3}\right)$; 127.8; 145.1; 158.5; 162.2; 163.9. Anal. Cald. for $\mathrm{C}_{20} \mathrm{H}_{18} \mathrm{~F}_{3} \mathrm{~N}_{5} \mathrm{O}_{2} \mathrm{~S}_{2}(481.51)$. Calculated (\%): C, 49.89; $\mathrm{H}, 3.77 ; \mathrm{N}, 14.54 ;$ Found (\%): C, 49.75; H, 3.62; N, 14.39.

5-(2-Fluorophenyl)-2,8-bis(ethylthio)-5,10-dihydropyrido[2,3-d:5,6- $d$ ] dipyrimidin-4,6(3H,7H)-dione (3q). White powder, m.p. $>300{ }^{\circ} \mathrm{C}$. IR, v/cm ${ }^{-1}: 3362 ; 3198 ; 2963 ; 2923 ; 2873 ; 1653 ; 1608 ; 1533 ; 1510 ; 1439 ; 1439 ; 1343 ; 1228 ; 758$. ${ }^{1} \mathrm{H}$ NMR (DMSO-d $)$ ), $\delta: 1.30$ (t, $6 \mathrm{H}, \mathrm{CH}_{3}, J=7.2 \mathrm{~Hz}$ ); 3.09 (q, 4H, CH, $\mathrm{J}=7.2 \mathrm{~Hz}$ ); 5.48 (s, $\left.1 \mathrm{H}, \mathrm{CH}\right) ; 6.8(\mathrm{br} . \mathrm{s}, 1 \mathrm{H}, \mathrm{NH})$; $6.98\left(\mathrm{dd}, 1 \mathrm{H}, \mathrm{Ar}-\mathrm{H}, J_{1}=11.2 \mathrm{~Hz}, J_{2}=8.4 \mathrm{~Hz}\right) ; 7.07-7.05(\mathrm{~m}, 2 \mathrm{H}, \mathrm{Ar}-\mathrm{H}) ; 7.19-7.17(\mathrm{~m}, 1 \mathrm{H}, \mathrm{Ar}-\mathrm{H}), 11.95(\mathrm{br} . \mathrm{s}, 2 \mathrm{H}, \mathrm{NH}-$ $\mathrm{C}=\mathrm{O}) .{ }^{13} \mathrm{C}$ NMR (DMSO-d $\left.\mathrm{d}_{6}\right), \delta_{\mathrm{C}}: 15.2 ; 24.3 ; 29.8 ; 92.6 ; 115.0\left(\mathrm{~d},{ }^{2} \mathrm{~J}_{\mathrm{C}-\mathrm{F}}=23.0 \mathrm{~Hz}\right) ; 124.0\left(\mathrm{~d},{ }^{4} \mathrm{~J}_{\mathrm{C}-\mathrm{F}}=2.0 \mathrm{~Hz}\right) ; 127.5\left(\mathrm{~d},{ }^{3} \mathrm{~J}_{\mathrm{C}-\mathrm{F}}\right.$ $=8.0 \mathrm{~Hz}) ; 127.6\left(\mathrm{~d},{ }^{2} J_{\mathrm{C}-\mathrm{F}}=16.8 \mathrm{~Hz}\right) ; 128.9\left(\mathrm{~d},{ }^{3} \mathrm{~J}_{\mathrm{C}-\mathrm{F}}=4.0 \mathrm{~Hz}\right) ; 158.0 ; 161.4 ; 161.5\left(\mathrm{~d},{ }^{1} \mathrm{~J}_{\mathrm{C}-\mathrm{F}}=244.0 \mathrm{~Hz}\right) ; 163.6$. $\mathrm{C}_{19} \mathrm{H}_{18} \mathrm{FN}_{5} \mathrm{O}_{2} \mathrm{~S}_{2}$ (431.51). Anal. Cald. for Calculated (\%): C, 52.89; H, 4.20; N, 16.23; Found (\%): C, 52.73; H, 4.08; N, 16.21 .

5-(2-Bromophenyl)-2,8-bis(ethylthio)-5,10-dihydropyrido[2,3-d:5,6- $d$ ] dipyrimidin-4,6(3H,7H)-dione (3r). White powder, m.p. > $300{ }^{\circ} \mathrm{C} . \mathrm{IR}, \mathrm{v} / \mathrm{cm}^{-1}: 3362 ; 3198 ; 2960 ; 2920 ; 2860 ; 1649 ; 1608 ; 1531 ; 1450 ; 1450 ; 1232 ; 800 .{ }^{1} \mathrm{H}$ NMR (DMSO-d 6 ), $\delta: 1.30\left(\mathrm{t}, \mathrm{J}=7.2 \mathrm{~Hz}, 6 \mathrm{H}, \mathrm{CH}_{3}\right) ; 3.11$ (br.s, 4H, CH$) ; 5.35(\mathrm{~s}, 1 \mathrm{H}, \mathrm{CH}) ; 6.88(\mathrm{~s}, \mathrm{br}, 1 \mathrm{H}, \mathrm{NH}) ; 7.09-7.06(\mathrm{~m}, 1 \mathrm{H}$, Ar-H); 7.27-7.25 (m, 2H, Ar-H); 7.47 (d, $1 \mathrm{H}, \mathrm{Ar}-\mathrm{H}, \mathrm{J}=8.0 \mathrm{~Hz}) ; 11.78(\mathrm{~s}, \mathrm{br}, 1 \mathrm{H}, \mathrm{NH}-\mathrm{C}=\mathrm{O}) ; 12.05$ (br. s, $1 \mathrm{H}, \mathrm{NH}-\mathrm{C}=\mathrm{O}) .{ }^{13} \mathrm{C}$ NMR (DMSO-d $), \delta_{C}: 15.1 ; 24.3 ; 35.5 ; 123.6 ; 127.4 ; 127.8 ; 129.5 ; 133.1 ; 140.4 ; 158.0 ; 158.1 ; 161.82 ; 161.84$. $\mathrm{C}_{19} \mathrm{H}_{18} \mathrm{BrN}_{5} \mathrm{O}_{2} \mathrm{~S}_{2}$ (492.41). Anal. Cald. for Calculated (\%): C, 46.34; H, 3.68; N, 14.22; Found (\%): C, 46.25; H, 3.58; N, 14.31 .

5-(4-Methoxyphenyl)-2,8-bis(ethylthio)-5,10-dihydropyrido[2,3-d:5,6- $\boldsymbol{d}^{\prime}$ ]dipyrimidin-4,6(3H,7H)-dion $\quad$ (3s). White powder, m.p. > $300^{\circ} \mathrm{C}$. IR, v/cm ${ }^{-1}: 3350 ; 3178 ; 2959 ; 2946 ; 1649 ; 1606 ; 1578 ; 1539 ; 1512 ; 1441 ; 1441 ; 1335 ; 1240 ; 1034 ;$ 833. ${ }^{1} \mathrm{H}$ NMR (DMSO-d $\mathrm{d}_{6}$ ), $\delta: 1.30\left(\mathrm{t}, 6 \mathrm{H}, \mathrm{CH}_{3}, J=7.2 \mathrm{~Hz}\right) ; 3.10\left(\mathrm{q}, 4 \mathrm{H}, \mathrm{CH}_{2}, J=7.2 \mathrm{~Hz}\right) ; 5.42(\mathrm{~s}, 1 \mathrm{H}, \mathrm{CH}) ; 3.71(\mathrm{~s}, 3 \mathrm{H}$, $\left.\mathrm{OCH}_{3}\right) ; 6.65$ (br. s, $\left.1 \mathrm{H}, \mathrm{NH}\right) ; 6.77(\mathrm{~d}, 2 \mathrm{H}, \mathrm{Ar}-\mathrm{H}, J=8.6 \mathrm{~Hz}) ; 6.92(\mathrm{~d}, 2 \mathrm{H}, \mathrm{Ar}-\mathrm{H}, J=8.6 \mathrm{~Hz}$ ); 11.93 (br. s, $2 \mathrm{H}, \mathrm{NH}-\mathrm{C}=\mathrm{O}) .{ }^{13} \mathrm{C}$ NMR (DMSO-d d $_{\text {) }} \delta_{c}: 15.2 ; 24.3 ; 33.2 ; 55.3 ; 93.4 ; 113.5 ; 128.0 ; 131.4 ; 157.2 ; 158.0 ; 162.2 ; 163.8$. Anal. Cald. for $\mathrm{C}_{20} \mathrm{H}_{21} \mathrm{~N}_{5} \mathrm{O}_{3} \mathrm{~S}_{2}$ (443.54). Calculated (\%): C, 54.16; H, 4.77; N, 15.79; Found (\%): C, 54.03; H, 4.68; N, 15.70.

5-(4-Formylphenyl)-2,8-bis(ethylthio)-5,10-dihydropyrido[2,3- $d: 5,6-d$ ] dipyrimidin-4,6(3H,7H)-dion $\quad$ (3t). White powder, m.p. $>300{ }^{\circ} \mathrm{C} . \mathrm{IR}, \mathrm{v} / \mathrm{cm}^{-1}: 3367 ; 3184 ; 2966 ; 2924 ; 1695 ; 1642 ; 1608 ; 1570 ; 1435 ; 1435 ; 1335 ; 1227,831 .{ }^{1} \mathrm{H}$ NMR (DMSO-d $\left.\mathrm{d}_{6}\right), \delta: 1.31\left(\mathrm{t}, J=7.2 \mathrm{~Hz}, 6 \mathrm{H}, \mathrm{CH}_{3}\right) ; 3.13-3.10\left(\mathrm{~m}, 4 \mathrm{H}, \mathrm{CH}_{2}\right) ; 5.53(\mathrm{~s}, 1 \mathrm{H}, \mathrm{CH}) ; 6.86(\mathrm{br} . \mathrm{s}, 1 \mathrm{H}, \mathrm{NH}) ; 7.25(\mathrm{~d}$, 
$2 \mathrm{H}, \mathrm{Ar}-\mathrm{H}, J=8.0 \mathrm{~Hz}$ ); 7.77 (d, 2H, Ar-H, J=8.0 Hz); 9.94 (s, 1H, CHO); 12.02 (br.s, 2H, NH-C=O). ${ }^{13} \mathrm{C}$ NMR (DMSO-d $)$, $\delta_{C}: 15.2 ; 24.3 ; 34.5 ; 92.6 ; 123.5 ; 127.8 ; 129.6 ; 134.2 ; 193.1 ; 164.7 ; 158.5 ; 147.9$. Anal. Cald. For $\mathrm{C}_{20} \mathrm{H}_{19} \mathrm{~N}_{5} \mathrm{O}_{3} \mathrm{~S}_{2}$ (441.53).Calculated (\%): C, 54.41; H, 4.34; N, 15.86; Found (\%): C, 54.58; H, 4.25; N, 15.71.

5-(Naphthalen-2-yl)-2,8-bis(ethylthio)-5,10-dihydropyrido[2,3-d:5,6- $d$ ] dipyrimidin-4,6(3H,7H)-dione (3u). White powder, m.p. $>300{ }^{\circ} \mathrm{C} . \mathrm{IR}, \mathrm{v} / \mathrm{cm}^{-1}: 3350 ; 3184 ; 2950 ; 2926 ; 2858 ; 1653 ; 1608 ; 1533 ; 1443 ; 1443 ; 1329 ; 1238 ; 814 ; 739 ;$ 708. ${ }^{1} \mathrm{H}$ NMR (DMSO-d $)$, $\delta: 1.33\left(\mathrm{t}, J=7.4 \mathrm{~Hz}, 6 \mathrm{H}, \mathrm{CH}_{3}\right) ; 3.14-3.12\left(\mathrm{~m}, 4 \mathrm{H}, \mathrm{CH}_{2}\right) ; 5.63(\mathrm{~s}, 1 \mathrm{H}, \mathrm{CH}) ; 6.83(\mathrm{br} . \mathrm{s}, 1 \mathrm{H}, \mathrm{NH}) ;$ $7.25(\mathrm{~d}, 1 \mathrm{H}, \mathrm{Ar}-\mathrm{H}, \mathrm{J}=8.6 \mathrm{~Hz}) ; 7.43-7.41(\mathrm{~m}, 2 \mathrm{H}, \mathrm{Ar}-\mathrm{H}) ; 7.46(\mathrm{~s}, 1 \mathrm{H}, \mathrm{Ar}-\mathrm{H}) ; 7.74(\mathrm{~d}, 1 \mathrm{H}, \mathrm{Ar}-\mathrm{H}, J=8.6 \mathrm{~Hz}) ; 7.84-7.77(\mathrm{~m}$, $2 \mathrm{H}, \mathrm{Ar}-\mathrm{H}$ ); 11.99 (br. s, $2 \mathrm{H}, \mathrm{NH}-\mathrm{C}=\mathrm{O}$ ). ${ }^{13} \mathrm{C}$ NMR (DMSO-d $)$ ), $\delta_{\mathrm{c}}: 15.2 ; 24.3 ; 34.2 ; 89.0 ; 124.0 ; 125.3 ; 126.0 ; 127.0 ; 127.4 ;$ 127.6; 127.9; 128.8; 131.8; 133.5; 137.7; 139.0; 158.5. Anal. Cald. for $\mathrm{C}_{23} \mathrm{H}_{21} \mathrm{~N}_{5} \mathrm{O}_{2} \mathrm{~S}_{2}$ (463.58). Calculated (\%): C, 59.59; H, 4.57; N, 15.11; Found (\%): C, 59.67; H, 4.62; N, 15.01 .

5-(Phenyl)-2,8-bis(ethylthio)-5,10-dihydropyrido[2,3-d:5,6- $d$ ]dipyrimidin-4,6(3H,7H)-dione (3v). White powder, m.p. $>300{ }^{\circ} \mathrm{C} . \mathrm{IR}, \mathrm{v} / \mathrm{cm}^{-1}: 3360 ; 3302 ; 3194 ; 2972 ; 2924 ; 2862 ; 1610 ; 1568 ; 1539 ; 1443 ; 1443 ; 1373 ; 1238 ; 773 ; 694 .{ }^{1} \mathrm{H} \mathrm{NMR}$ (DMSO-d $\mathrm{d}_{6}$ ), $\delta: 1.31$ (t, $\left.6 \mathrm{H}, \mathrm{CH}_{3}, J=7.0 \mathrm{~Hz}\right) ; 3.10$ (q, 4H, $\left.\mathrm{CH}_{2}, J=7.0 \mathrm{~Hz}\right) ; 5.48(\mathrm{~s}, 1 \mathrm{H}, \mathrm{CH}) ; 6.77$ (br. s, $\left.1 \mathrm{H}, \mathrm{NH}\right) ; 7.03(\mathrm{~d}$, $2 \mathrm{H}, \mathrm{Ar}-\mathrm{H}, J=7.6 \mathrm{~Hz}$ ); 7.1 (t, $1 \mathrm{H}, \mathrm{Ar}-\mathrm{H}, J=7.0 \mathrm{~Hz}$ ); 7.20 (t, $2 \mathrm{H}, \mathrm{Ar}-\mathrm{H}, J=7.4 \mathrm{~Hz}) ; 11.82$ (br. s, $2 \mathrm{H}, \mathrm{NH}-\mathrm{C}=\mathrm{O}) .{ }^{13} \mathrm{C} \mathrm{NMR}$ (DMSO-d ${ }_{6}$ ), $\delta_{C}: 15.2 ; 24.3 ; 33.9 ; 93.0 ; 125.3 ; 127.0 ; 139.8 ; 128.0 ; 158.1 ; 161.2 ; 162.8$. Anal. Cald. for $\mathrm{C}_{19} \mathrm{H}_{19} \mathrm{~N}_{5} \mathrm{O}_{2} \mathrm{~S}_{2}$ (413.52). Calculated (\%): C, 55.19; H, 4.63; N, 16.94; Found (\%): C, 55.10; H, 4.78; N, 16.75.

\section{CONCLUSION}

In summary, we have developed a simple and efficient protocol for the synthesis of new derivatives of dihydropyridodipyrimidines in the mixed solvent of glacial acetic acid and DMF $(1: 1, \mathrm{v}: \mathrm{v})$ under ultrasonic irradiation. The reaction induced by ultrasound offered better yields and much lower reaction times than the conventional heating. The easy work of the products, mild reaction condition and cleaner reaction are the notable features of this protocol.

\section{ACKNOWLEDGMENTS}

The authors are grateful to the Research Council of Islamic Azad University, Rasht Branch for partial financial support of this work.

\section{REFERENCES}

[1] Sanghvi, Y. S., Larson, S. B., Matsumoto, S. S., Nord, L. D., Smee, D. F., Willis, R. C., Avery, T. H., Robins, R. K., Revankar, G. R. 1989. Antitumor and antiviral activity of synthetic alpha- and beta-ribonucleosides of certain substituted pyrimido[5,4-d]pyrimidines: a new synthetic strategy for exocyclic aminonucleosides. J. Med. Chem. 32 , 629-637.

[2] Nizamuddin-Mishra, M., Srivastava, M. K., Khan, M. H. 2001. Synthesis and fungicidal activity of 3,6,9-triaryl-2thioxothiazolo[4,5- $d][1,3,4]$ oxadiazolo[2,3-b]pyrimidine and 3,10-diaryl-2-thioxothiazolo[4,5- $d$-pyrimido [2,1-b] pyrimidines. Indian J. Chem. 40, 49-53.

[3] Ram, V. J., Goel, A., Sarkhel, S., Maulik, P. R. 2002. A Convenient Synthesis and Hepatoprotective Activity of Imidazo[1,2-c]pyrimido[5,4-e]pyrimidine, Tetraazaacenaphthene and Tetraazaphenalene from Cyclic Ketene Aminals Through Tandem Addition-Cyclization Reactions. Bioorg. Med. Chem. 10, 1275-1280.

[4] Tenser, R. B., Gaydos, A., Hay, K. A. 2001. Inhibition of herpes simplex virus reactivation by dipyridamole. Antimicrob. Agents Chemother. 45, 3657-3659.

[5] Clercq, E. D., Beraaerts, R. Specific phosphorylation of 5-ethyl-2'-deoxyuridine by herpes simplex virus-infected cells and incorporation into viral DNA. 1987. J. Biol. Chem. 262, 14905-14911.

[6] Gready, J. E., McKinlay, C., Gebauer, M. G. 2003. Synthesis of quaternised 2-aminopyrimido[4,5-d]pyrimidin-4(3H)ones and their biological activity with dihydrofolate reductase. Eur. J. Med. Chem. 38, 719-728.

[7] Parish, H. A., Gilliom, R. D., Purcell, W. P., Browne, R. K., Sprik, R. F., White, H. D. 1982. Syntheses and diuretic activity of 1,2-dihydro-2-(3-pyridyl)-3H-pyrido[2,3-d]pyrimidin-4-one and related compounds. J. Med. Chem. 25, 98-102.

[8] Hurlbert, B. S., Valenti, B. F. 1968. Studies on Condensed Pyrimidine Systems. XXIV. The Condensation of 2,4,6Triaminopyrimidine with Malondialdehyde Derivatives. J. Med. Chem. 11, 708-710.

[9] El-Gazzar, A. B. A., Hafez, H. N. 2009. Synthesis of 4-substituted pyrido[2,3-d]pyrimidin-4(1H)-one as analgesic and anti-inflammatory agentsBioorg. Med. Chem. Lett. 19, 3392-3397.

[10] Bouzard, D., in, Antibiotics and Antiviral Compounds (Krohr, K., Kirst, H. A., and Maag, H., Eds) p. 168. VCH, Weinheim, 1993.

[11] Youssif, S., El-Bahaie, S., Nabih, E. J. 1999. A facile one-pot synthesis of pyrido[2.3-d]pyrimidines and pyrido[2,3-d 6,5-d']dipyrimidines. Chem. Research (S), 112-113.

[12] Kretzschmar, E. 1980. Derivatives of 4-oxo-3,4-dihydropyrido[2,3-d]pyrimidine. Pharmazie, 35, 253-256. 
[13] Piper, J. R., McCaleb, G. S., Montgomery, J. A., Kisliuk, R. L., Sirotanks, F. M. 1986. Synthsis and Antifolate Activity of 5-Methyl-5-Deaza Analogs of Aminopterin, MTX, Folic Acid and N10-Methyl Folic Acid. J. Med. Chem. 29, 10801087.

[14] Furuya, S., Ohtaki, T. 1994. Eur. Pat. Appl., EP 0608565 A1; Chem. Abstr. 121, 205395.

[15] Heber, D., Heers, C., Ravens, U. 1993. Positive inotropic activity of 5-amino-6-cyano-1,3-dimethyl-1,2,3,4tetrahydropyrido[2,3-d]pyrim idine-2,4-dione in cardiac muscle from guinea-pig and man. Part 6: Compounds with positive inotropic activity. Pharmazie, 48, 537.

[16] Cordeu, L., Cubedo, E., Bandrés, E., Rebollo, A., Sáenz, X., Chozas, H., Domínguez, M. V., Echeverría, M., Mendivil, B., Sanmartin, C., Palop, J. A., Font, M., Foncillas, G. 2007. Bioorg. Med. Chem. 15, 1659-1669.

[17] Ghorab, M. M., Hassan, A. Y. 1998. Synthesis and antibacterial properties of new dithienyl containing pyran, , pyrano[2,3-b] pyridine, pyrano[2,3-d]pyrimidine and pyridine derivatives. Phosphorus Sulfur Silicon Relat Elem. 141, $251-261$.

[18] Sakuma, Y., Hasegawa, M., Kataoka, K., Hoshina, K., Yamazaki, N., Kadota, T., Yamaguchi, H. 1991. PCT Int. Appl. 1989, WO 91/05785; Chem. Abstr. 115, 71646.

[19] Quintela, J. M., Peinador, C., Botana, L., Estevez, M., Riguera, R. 1997. Synthesis and antihistaminic activity of 2guanadino-3-cyanopyridines and pyrido[2,3-d]-pyrimidines. Bioorg. Med. Chem. 5, 1543-1553.

[20] Nasr, M. N., Gineinah, M. M. 2002. Pyrido [2, 3-d]pyrimidines and pyrimido[5', 4':5, 6]pyrido[2, 3- $d$ ]pyrimidines as new antiviral agents: synthesis and biological activity. Arch. Pharm. 335, 289-295.

[21] Hirota, K., Huang, J., Sajiki, H., Maki, Y. 1986. Pyrimidines. Part 57. A Versatile Synthesis of Pyrimido[4,5d]pyrimidine-2,4,5-trione Derivatives. Heterocycles, 24, 2293-2298.

[22] Niess, R., Robins, R. K. 1970. A new synthesis of the pyrimido[4,5-d] pyrimidine ring. Preparation of pyrimido[4,5- $d$ ] pyrimidine-2,4,5,7-tetrone. J. Heterocycl. Chem. 7, 243-244.

[23] Hirota, K., Kitade, H., Sajiki, H., Maki, Y . 1984. A Facile Synthesis of 7-Substituted Pyrimido[4,5-d]-pyrimidine-2,4diones. Synthesis. 589-590.

[24] Gohain, M., Prajapati, D., Gogoi, B. J., Sandhu, J. S. 2004. A Facile Microwave Induced One-Pot Synthesis of Novel Pyrimido[4,5-d]pyrimidines and Pyrido[2,3-d]pyrimidines under Solvent-Free Conditions. Synlett, 1179-1182.

[25] Quiroga, J., Cisneros, C., Insuasty, B., Abonia, R. 2006. Microwave-assisted three-component synthesis and in vitro antifungal evaluation of 6-cyano-5,8-dihydropyrido[2,3-d]pyrimidin-4(3H)-ones. J. Heterocycl. Chem. 43, $299-306$.

[26] Agarwal, A., Chauhan, P. M. S. 2005. Solid supported synthesis of structurally diverse dihydropyrido[2,3-d]pyrimidines using microwave irradiation. Tetrahedron Lett. 46, 1345-1348.

[27] Tu, S., Cao, L., Zhang, Y., Shao, Q., Zhou, D., Li, C. 2008. An efficient synthesis of pyrido[2,3-d]pyrimidine derivatives and related compounds under ultrasound irradiation without catalyst . Ultrason. Sonochem. 15, 217-221.

[28] Parajapati, D., Thakur, A. J. 2005. Studies on 6-[(dimethylamino)methylene]aminouracil: a facile one-pot synthesis of novel pyrimido[4,5-d]pyrimidine derivatives. Tetrahedron Lett. 46, 1433-1436.

[29] Mahdavinia, G. H., Rostamizadeh, S., Amani, A. M., Emdadi, Z. 2009. Ultrasound-promoted greener synthesis of aryl14-H-dibenzo[a,j]xanthenes catalyzed by $\mathrm{NH}_{4} \mathrm{H}_{2} \mathrm{PO}_{4} / \mathrm{SiO}_{2}$ in water. Ultrason. Sonochem. 16, 7-10.

[30] Cravotto, G., Cintas, P. 2006. Power ultrasound in organic synthesis: moving cavitational chemistry from academia to innovative and large-scale applications. Chem. Soc. Rev. 35, 180-196.

[31] Mason, T. J., Lorimer, J. P. 2002. Applied Sonochemistry, The Uses of Power Ultrasound in Chemistry and Processing, Wiley $\mathrm{VCH}$, Verlag $\mathrm{GmbH}$.

[32] Mantu, D., Moldoveanu, C., Nicolescu, A., Deleanu, C., Mangalagiu, I. I. 2009. A facile synthesis of pyridazinone derivatives under ultrasonic irradiation. Ultrason. Sonochem. 16, 452-454.

[33] Li, J. -T., Yin, Y., Li, L., Sun, M. -X. 2010. A convenient and efficient protocol for the synthesis of 5-aryl-1,3diphenylpyrazole catalyzed by hydrochloric acid under ultrasound irradiation. Ultrason. Sonochem. 17, 11-13.

[34] Pizzuti, L., Martins, P. L. G., Ribeiro, B. A., Quina, F. H., Pinto, E., Flores, A. F. C., Venzke, D., Pereira, C. M. P. 2010. Efficient sonochemical synthesis of novel 3,5-diaryl-4,5-dihydro-1H-pyrazole-1-carboximidamides. Ultrason. Sonochem. 17, 34-37.

[35] Ni , C. -L., Song, X. -H., Yan, H., Song, X. -Q., Zhong, R. -G. 2010. Improved synthesis of diethyl 2,6-dimethyl-4aryl-4H-pyran-3,5-dicarboxylate under ultrasound irradiation. Ultrason. Sonochem. 17, 367-369. 
[36] Bazgir, A., Ahadi, S., Ghahremanzadeh, R., Khavasi, H. R., Mirzaei, P. 2010. Ultrasound-assisted one-pot, threecomponent synthesis of spiro[indoline-3,4'-pyrazolo[3,4-b]pyridine]-2,6'(1'H)-diones in water. Ultrason. Sonochem. 17, 447-452.

[37] Zhang, Z. -H., Li, J.-J., Li, T. -S. 2008. Ultrasound-assisted synthesis of pyrroles catalyzed by zirconium chloride under solvent-free conditions. Ultrason. Sonochem. 15, 673-676.

[38] Khosropour, A. R. 2008. Ultrasound-promoted greener synthesis of 2,4,5-trisubstituted imidazoles catalyzed by $\mathrm{Zr}(\mathrm{acac})_{4}$ under ambient conditions. Ultrason. Sonochem. 15, 659-664.

[39] Mamaghani , M., Shirini, F., Mahmoodi, N. O., Azimi-Roshan, A., Hashemlou, H. 2013. A green, efficient and recyclable Fe ${ }^{+3} @ K 10$ catalyst for the synthesis of bioactive pyrazolo[3,4-b]pyridin-6(7H)-ones under " on water" condition. J. Mol. Struct. 1051, 169-176.

[40] Hossein Nia, R., Mamaghani, M., Tabatabaeian, K., Shirini, F., Rassa, M. 2012. An expeditious regioselective synthesis of novel bioactive indole-substituted chromene derivatives via one-pot three-component reaction. Bioorg. Med. Chem. Lett. 22, 5956-5960.

[41] Mamaghani, M., Loghmanifar, A., Taati, R. 2011. An efficient one-pot synthesis of new 2-imino-1,3-thiazolidin-4-ones under ultrasonic conditions. Ultrason. Sonochem. 18, 45-48.

[42] Nikpassand, M., Mamaghani, M., Shirini, F., Tabatabaeian, K., 2010. A convenient ultrasound-promoted regioselective synthesis of fused polycyclic 4-aryl-3-methyl-4,7-dihydro-1 H-pyrazolo[3,4-b]pyridines. Ultrason. Sonochem. 17, 301-305.

[43] Crepaldi, P., Cacciari, B., Bonache, M. C., Spalluto, G., Varani, K., Borea, P. A., Kügelgen, I. V., Hoffmann, K., Pugliano, M., Razzari, C., Cattaneo, M. 2009. 6-Amino-2-mercapto-3H-pyrimidin-4-one derivatives as new candidates for the antagonism at the $\mathrm{P}_{2} \mathrm{Y}_{12}$ receptors. Bioorg. Med. Chem. 17, 4612-4621. 